\title{
Spin-canting and weak ferromagnetism in two novel 1D alternating chains with single cis-end-to-end azido bridges
}

\author{
SUN HaoLing ${ }^{1 *}$, XIONG Jin $^{1}, \mathrm{JI}_{\text {ShaoFei }}{ }^{1}$, ZHEN Xue ${ }^{1}, \mathrm{JIA} \mathrm{Li}^{1}$, LIU Tao $^{2}$ \\ 1 Department of Chemistry, Beijing Normal University, Beijing 100875, China \\ 2 State Key Laboratory of Fine Chemicals; Dalian University of Technology, Dalian 116012, China \\ *Corresponding authors (email: haolingsun@bnu.edu.cn)
}

Received January 31, 2012; accepted February 26, 2012; published online April 13, 2012

全文见: Sun HL, Xiong J, Ji SF, Zhen X, Jia L, Liu T. Spin-canting and weak ferromagnetism in two novel 1D alternating chains with single cis-end-to-end azido bridges. Sci China Chem, 2012, 55(6): 1031-1036

\begin{abstract}
By combining trans-4-styrylprydine (tsp) with azide system, we obtained two rare 1D cis-EE-azido-bridged compounds of $\left[\mathrm{M}_{2}\left(\mathrm{~N}_{3}\right)_{4}(\mathrm{tsp})_{4}\left(\mathrm{H}_{2} \mathrm{O}\right)_{2}\right][\mathrm{M}=\mathrm{Mn}$ (1), Co (2)]. The magnetic study reveals that metal ions are anti-ferromagnetically coupled by cis-EE-azido-bridges and they show weak ferromagnetism below $4.0 \mathrm{~K}(\mathbf{1})$ and $4.2 \mathrm{~K}$ (2) due to the spin-canting caused by asymmetric magnetic interaction.
\end{abstract}

Keywords: single cis-end-to-end azide, 1D complex, anti-ferromagnetic coupling, spin-canting, weak ferromagnetism

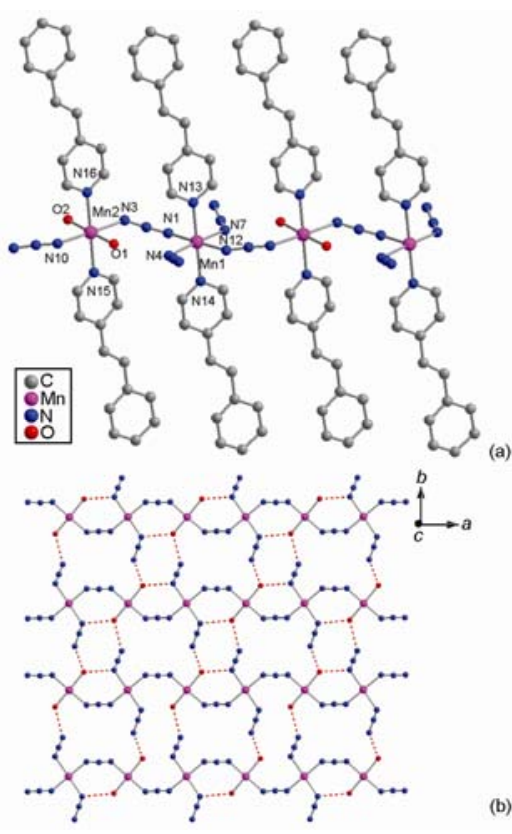

(a) The fragment of 1D chain in $\mathbf{1}$ and 2; (b) supramolecular layers formed by $\mathrm{O}-\mathrm{H} \cdots \mathrm{N}$ hydrogen bonds.

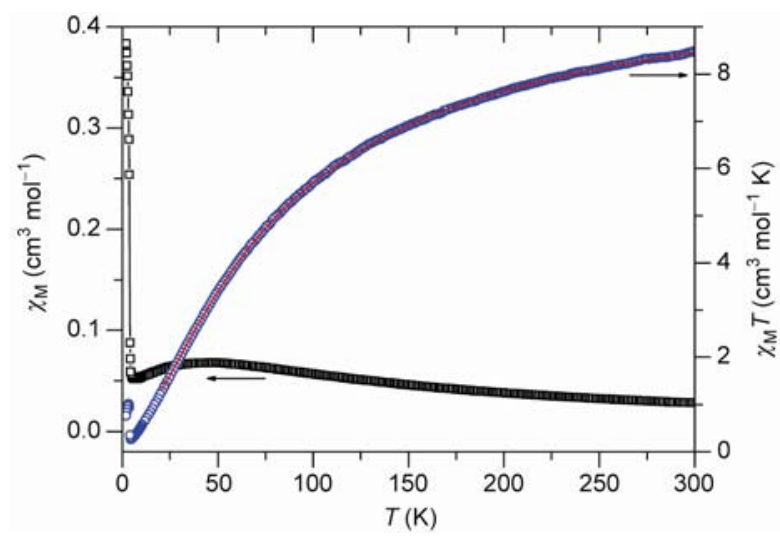

Temperature dependences of $\chi_{\mathrm{M}}$ and $\chi_{\mathrm{M}} T$ for a polycrystalline sample of $\mathbf{1}$. The line across $\chi_{\mathrm{M}} T$ curve represents the best fit by $1 \mathrm{D}$ Fisher mode. 


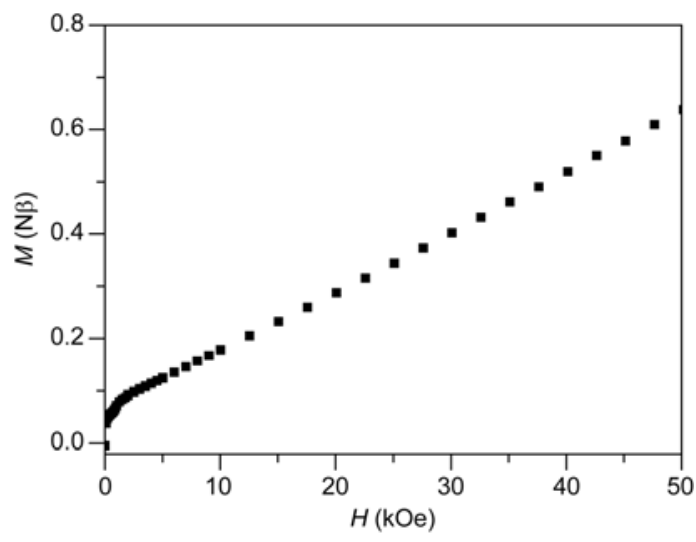

Isothermal magnetization plots for $\mathbf{1}$ at $1.9 \mathrm{~K}$.

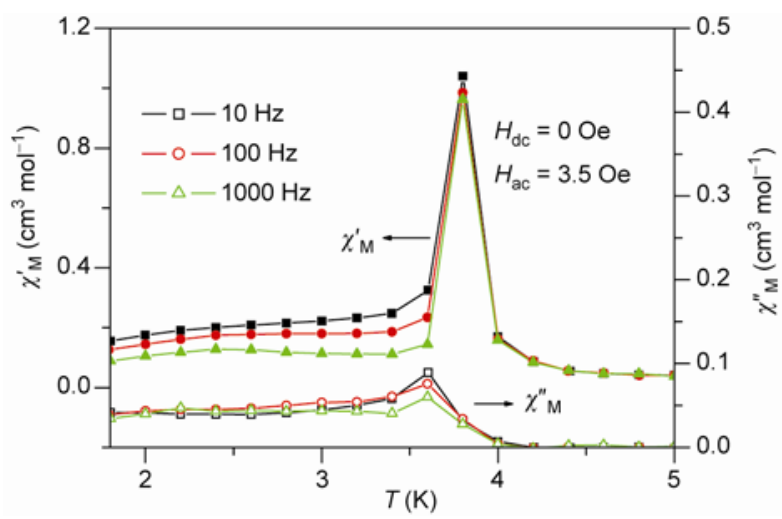

The zero-field $a c$ magnetic signals of $\mathbf{1}$.

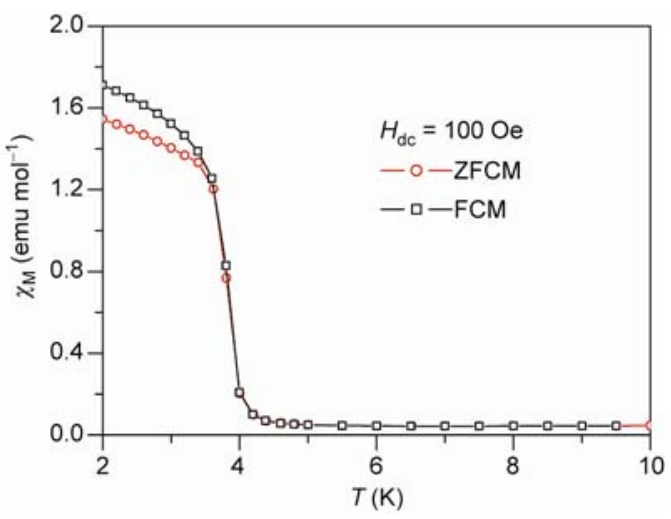

Zero-field cooled magnetization (ZFCM) and field-cooled magnetization (FCM) for $\mathbf{1}$ under $100 \mathrm{Oe}$.

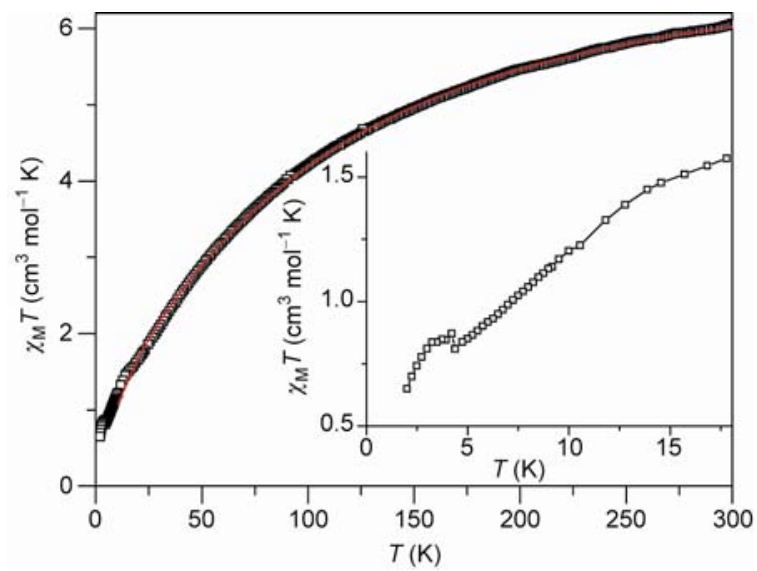

Temperature dependences of $\chi_{\mathrm{M}} T$ for a polycrystalline sample of $\mathbf{2}$. The solid line represents the best fitting results of single-ions magnetic behavior of $\mathrm{Co}^{2+}$ with the consideration of molecular field. 